\title{
La webquest como recurso didactico para la promocion de aprendizaje significativo en ciencias naturales y exactas
}

Rafael Silva Córdova rsilvacordova@gmail.com 0000-0003-2185-0285 Universidad de Playa Ancha, Valparaiso, Chile.

Ester Lopez Donoso elopez@upla.cl

0000-0001-6846-6761

Universidad de Playa Ancha, Valparaiso

Chile.

Juan Carlos Medina Magdaleno uancarlosm29@gmail.com 0000-0002-8682-9308

Universidad de Playa Ancha, Valparaíso, Chile.

\section{RESUMEN}

La Webquest es un tipo de actividad didáctica basada en presupuestos constructivistas del proceso enseñanza - aprendizaje. La presente investigación, en el contexto de la innovación, propone una experiencia didáctica para a tres grupos de profesores, para diseñar, elaborar y poner en práctica webquest para la enseñanza de las ciencias. Se orienta en una metodología indagatoria guiada y que promueva aprendizaje significativo, y que mejore los rendimientos académicos. La propuesta metodológica de enseñanza se sustenta teóricamente en el aprendizaje significativo de Ausubel, en desarrollos cognitivos de Piaget zona de desarrollo próximo de Vygotsky y el trabajo cooperativo de Jonhson y Jonhson. La investigación es de tipo experimental, esencialmente cuantitativa, con un estudio exploratorio, descriptivo. Los resultados aseguran un avance importante entre el pretest y postest de cada grupo de trabajo, con un producto tangible que puede ser utilizado en las aulas de ciencias.
\end{abstract}

PALABRAS CLAVES: Webquest. Aprendizaje significativo. Rendimiento académico. Asimilación. Metodología de enseñanza indagatoria. 


\section{INTRODUCCION}

La innovación educativa es un término recurrente en el discurso educativo actual, y se presenta como un elemento central en el proceso enseñanza aprendizaje, pero existe una fuerte problemática en la conceptualización de la innovación educativa, la cual se manifestaba en cuatro indicadores:

- La falta de un marco teórico suficientemente desarrollado y compartido;

- Diferentes denominaciones del término;

- La reducción del contenido conceptual de la innovación educativa al volverse sinónimo de innovación tecnológica en educación;

- La subordinación de la innovación a la mejora continua.

Debe quedar con claridad y convicción que la innovación en educación está directamente relacionada con el mejoramiento del proceso enseñanza aprendizaje. La educación en ciencias, tiene como objetivo hacer que el alumno aprenda a compartir significados en el contexto de las ciencias, o sea, interpretar el mundo desde el punto de vista de las ciencias, manejar algunos conceptos, leyes y teorías científicas, abordar problemas razonando científicamente, identificar aspectos históricos, sociales y culturales de las ciencias. El entrenamiento de un científico debe incluir la educación en ciencias, pero no a la inversa. La educación en ciencias no implica poner al alumno en un laboratorio, ni transformarlo en un especialista en resolución de problemas, o un futuro investigador. No es su misión.

La producción de conocimiento en educación en ciencias es la búsqueda de respuestas a preguntas sobre enseñanza, aprendizaje, currículum y contexto educativo, así como el profesorado de ciencias y su formación permanente, dentro de un cuadro epistemológico, teórico y metodológico consistente y coherente en el cual el contenido específico de las ciencias está siempre presente. La investigación básica en educación en ciencias a enfocado sus estudios a factores de desarrollo, debilidades, recomendaciones de que investigar y a la formación de profesores.

Las tendencias actuales en investigaciones en ciencias son relativas a la evaluación de aprendizajes, al uso de las nuevas tecnologías, a la formación de profesores de ciencias. Sus principales debilidades las dirige a la falta de líneas progresivas de investigaciones, que carecen de marco teórico, metodológicos y epistemológicos coherentes, a la poca transferencia al aula de conocimiento producido por las investigaciones, al uso de los enfoques cualitativos con la etiqueta que "todo vale", falta de una visión más compleja de los procesos de aprendizaje que no son sólo cognitivo. En las recomendaciones para las investigaciones en educación en ciencias, Moreira (2000) propone mejorar la calidad de las investigaciones en términos de preguntas, fundamentación teórica, metodológica y epistemológica, preocupándose de las cuestiones foco de estudio, la incorporación de profesores a grupos de investigación, generar líneas de trabajo progresivas. Las universidades y sistemas de educación secundaria y primaria deberían promover medios y valorar la investigación en enseñanza de las ciencias, para que los profesores no universitarios puedan participar de los grupos y 
debe estar presente en el currículo de la formación inicial de profesores de ciencias.

Una forma de preparar a los estudiantes para el futuro es implementar el aprendizaje apoyado en Internet que utiliza con propósitos educativos la información publicada actualmente en la Red. Los estudiantes tienen hoy, como nunca antes, la posibilidad de consultar fuentes primarias de información y conocer diferentes puntos de vista sobre un mismo hecho. Esta situación contrasta con el uso de los libros de texto tradicionales que ofrecen solo la visión de su autor con sus posibles sesgos. El objetivo fundamental de la Webquest es lograr que los estudiantes hagan buen uso del tiempo y se enfoquen en la utilización de la información más que en buscarla.

La presente investigación propone un taller de uso de TIC para la enseñanza de las ciencias, es parte de la formación del programa de magister en enseñanza de las ciencias de la Universidad de Playa Ancha, acreditado por siete años. El taller está estructurado en el uso de la Webquest como recurso de enseñanza de las ciencias para mejora los rendimientos académicos y su aprendizaje, donde las actividades a cumplir deben promover aprendizaje significativo en sus alumnos. El curso taller debe terminar con un producto realizado en grupo que este orientado a ser utilizado en su realidad en el aula.

Las buenas Webquest provocan procesos cognitivos superiores (transformación de información de fuentes y formatos diversos, comprensión, comparación, elaboración y contraste de hipótesis, análisis-síntesis, creatividad, etc.). Para que los alumnos usen estas funciones superiores de la cognición, las Webquest utilizan "andamios cognitivos", un concepto muy relacionado con el de Zona de Desarrollo Próxima de Vigotsky. Se trata de estrategias para ayudar los estudiantes a organizar la información en unidades significativas, analizarla y producir respuestas nuevas. En la elaboración de las Webquest cada estudiante desempeña un rol específico en el seno de un grupo que debe coordinar sus esfuerzos para resolver una tarea o producir un producto. Comprender algo para explicarlo posteriormente a los compañeros implica normalmente un esfuerzo mayor del necesario para salir con éxito de las tareas escolares tradicionales, que finalizan con algún tipo de prueba de evaluación. Es más, en el grupo todo el mundo es necesario: las Webquest refuerzan la autoestima de los estudiantes porque promueven la cooperación y la colaboración entre los ellos para resolver una tarea común. Como destaca Cabero (1999), lo significativo en el trabajo colaborativo no es la simple existencia de interacción e intercambio de información entre los miembros del grupo, sino su aprendizaje sea adquirido por todo los integrantes del grupo. No se refiere, por tanto, la simple sumatoria de intervenciones, sino a la interacción conjunta para alcanzar objetivos previamente determinados. La estructura de una webquest contiene seis pasos bien definidos.

\section{Introducción}

2.Tarea

3.Proceso

4. Recursos

5.Evaluación 
La misión de educar es facilitar a docentes herramientas para enseñar en la sociedad del conocimiento. La visión de educar es aplicar las Tecnologías de la Información y la Comunicación a la solución de los problemas prioritarios de la educación. Por tal motivo, las actividades que se proponen dan lugar a:

- Poner a disposición de los docentes una técnica que facilite la integración de internet a la praxis diária;

- Ofrecer una metodología práctica, fácil y eficiente para trabajar con internet;

- Brindar actividades motivadoras, creativas, teniendo a la red como recurso essencial;

- Evitar vicios del uso educativo de internet, como el vagabundeo y el copy-paste.

Muchas veces se piensa que todo lo que se puede hacer con la computadora se puede hacer en el cuaderno de clases. Las webquest son actividades para realizar exclusivamente con la red, y favorecer la posibilidad de que los alumnos tengan acceso a contenidos interesantes, de calidad y actualizados. Internet brinda una gran cantidad de información, y el uso de esta metodología es una manera de aprovecharla. Los alumnos accederán a documentos de primera mano, fuentes primarias, etc., a los que sería muy difícil llegar de otra manera. No es la única ni principal manera de trabajar para lograr el desarrollo del pensamiento crítico de los alumnos, pero sí una nueva estrategia de este momento que brinda un valor agregado más a los recursos de nuestros docentes, y permite la transformación y la construcción del conocimiento. Para clarificar mejor el sustento teórico en que se basa la investigación se muestra en el esquema 1.

Esquema 1 - Teorías en que se sustenta la investigación

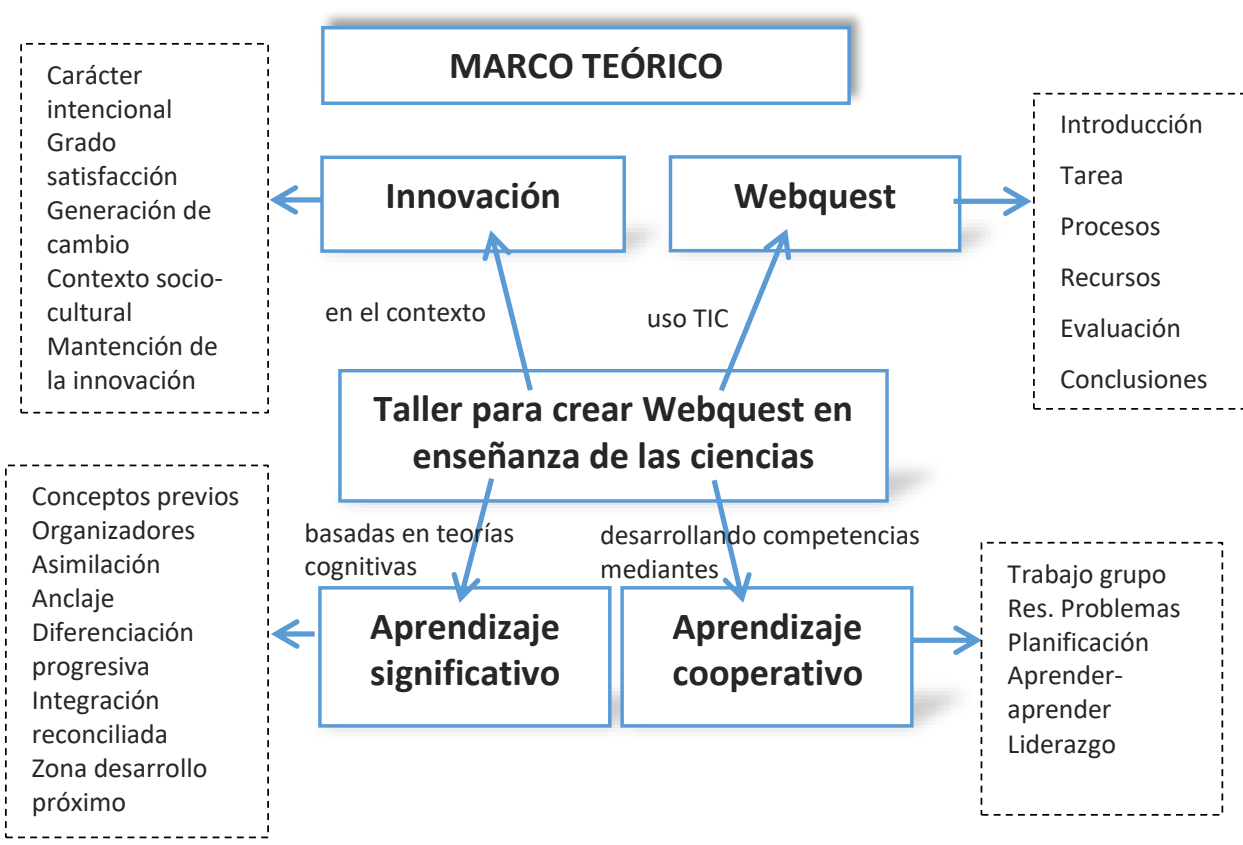

Fuente: Elaboracíón propia. 
¿Cuál es el rendimiento y el aprendizaje de un taller que desarrolla una metodología Webquest?

¿Cuáles son los aportes, en productos pertinentes y situados, del taller de Webquest a la enseñanza de las ciencias?

Para poder dar respuestas a las preguntas anteriores se plantean los siguientes objetivos generales:

Capacitar a los alumnos del taller sobre el uso de la Webquest como recurso didáctico de aprendizaje, especialmente que sea significativo.

Capacitar a los alumnos del taller con los recursos tecnológicos necesarios que utilizan una Webquest.

Capacitar a los alumnos del taller en el diseño, y elaboración de una Webquest que responda a las necesidades del sistema educativo.

\section{METODOLOGIA}

La metodología con que se resuelve el problema de investigación es cuantitativa y experimental, en particular un pre experimento, con pretest y postest.

El tipo de estudio, es descriptivo y comparativo.

El diseño de la investigación se muestra en el siguiente esquema 2.

Esquema 2 - Diseño de la investigación

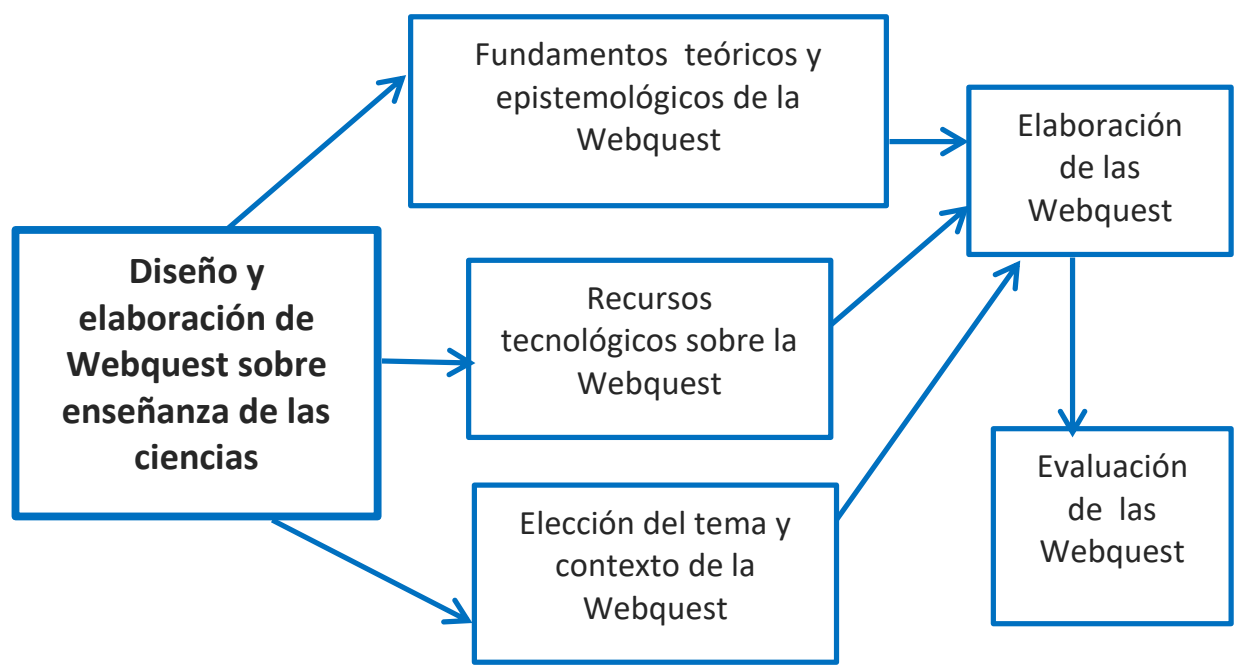

Fuente: Elaboración propia.

La variable taller de uso de TIC, mediante una metodología Webquest, se puede definir a través del siguiente esquema 3. 
Esquema 3 - Definición de la variable, taller de creación de Webquest en ciencias

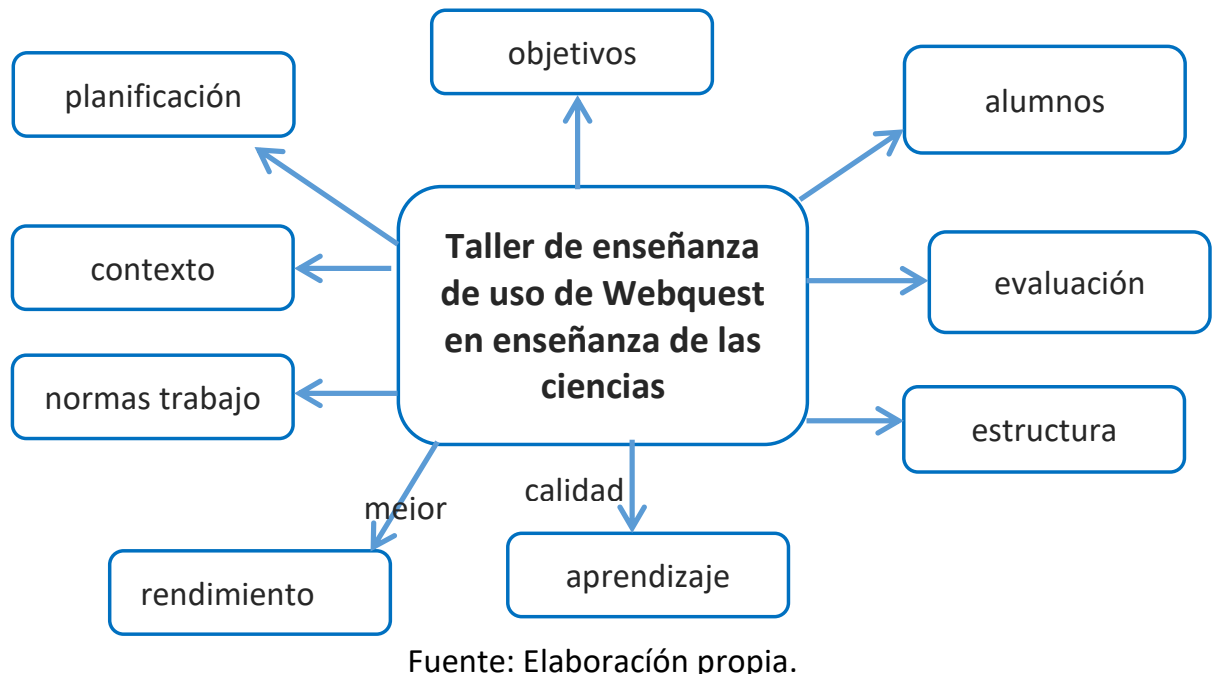

El rendimiento es el resultado del curso taller estos tres últimos años, medido mediante el proceso de evaluación (talleres, rúbricas, conceptos)

El aprendizaje como efecto que provoca la aplicación de la propuesta de enseñanza o taller de creación de Webquest, se han utilizados los siguientes medios:

Esquema 4 - Aprendizaje obtenido de cumplir con los objetivos del taller

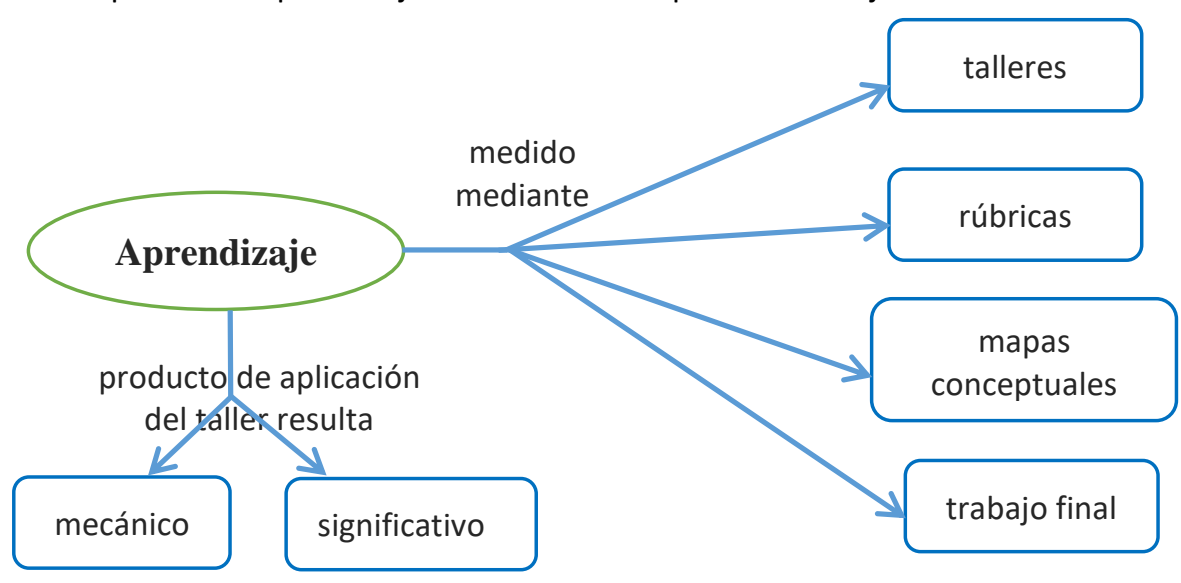

Fuente: Elaboracíón propia.

Productos del taller de diseño y elaboración de Webquest corresponde a la cantidad de webquest desarrollados por los alumnos.

Los instrumentos que evalúan las variables son: (1) la planificación que mide el cumplimiento de la metodología propuesta, (2) pretest y postest que mide el cumplimiento de los objetivos a lograr, (3) rúbricas sobre lo realizado en cada sesión, (4) talleres de resolución de problemas, (5) mapas conceptuales, (6) rúbrica para evaluar los productos realizado por cada alumno.

La muestra corresponde a 19 alumnos el 2015, 20 alumnos el 2016 y 18 alumnos el 2017. 


\section{ANALISIS Y DISCUSION DE RESULTADOS}

Los resultados sobre el rendimiento académico como nivel de conocimiento adquirido producto del aprendizaje de los contenidos de taller de creación de Webquest como recurso didáctico, se muestran en la tabla 1 con escala de evaluación de 1,0 a 7,0.

Tabla 1 - Rendimientos de los talleres Webquest

\begin{tabular}{ccc} 
Trabajo webquest & Promedio & Desv. Estándart $\sigma$ \\
& & \\
Año 2015 & 5,9 & 0,97 \\
Año 2016 & 6,1 & 0,89 \\
Año 2017 & 5,9 & 0,76 \\
\hline
\end{tabular}

Fuente: Elaboracíón propia.

Cuya representación gráfica se muestra a continuación

Gráfico 1 - Evaluación de Webquest según años

\section{EVALUACION DE TRABAJO WEBQUEST (POR AÑOS)}

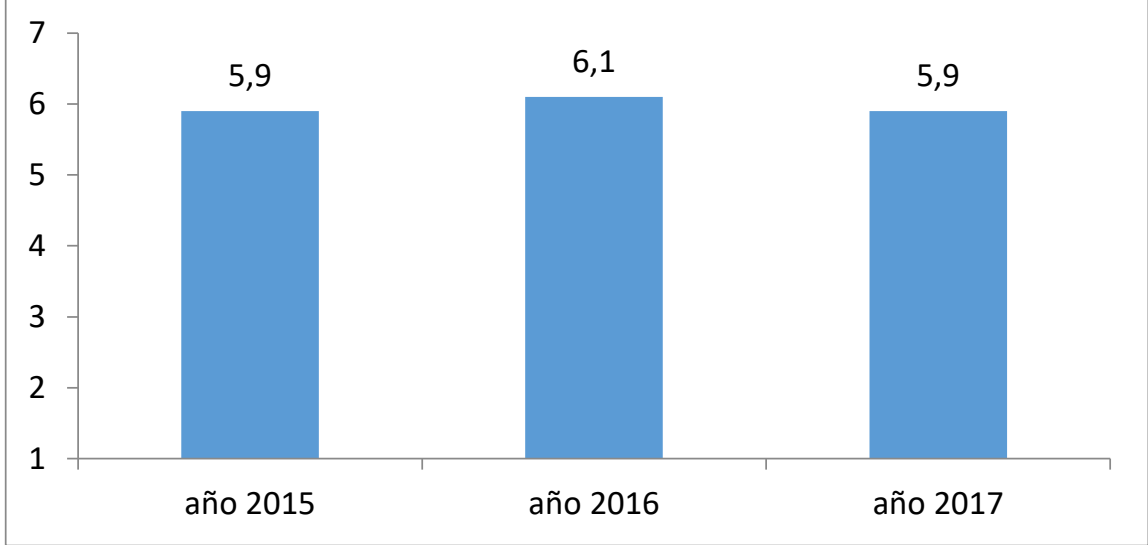

Fuente: Elaboracíón propia.

Se observa que no existe una diferencia significativa de acuerdo a los años en que se realiza el taller que se propone. Sin embargo se aprecia que los rendimientos de logros son importantes.

Respecto a los aprendizajes de los alumnos del taller, se puede señalar que durante el desarrollo de él, los alumnos construían aprendizaje significativo sobre el uso de la Webquest como recurso didáctico para el proceso enseñanza aprendizaje, situación verificada por mapas conceptuales (diferenciación progresiva) y por las aplicaciones de los conceptos en situaciones pertinentes (reconciliación integradora).

En cuanto a la experimentación se utilizó un pretest y un postest, cuyos resultados se muestran a continuación. 
Tabla 2 - Resultados del rendimiento entre pretest y postest

\begin{tabular}{cccc} 
& Promedio & $\begin{array}{c}\text { Desviación } \\
\text { stándart } \boldsymbol{\sigma}\end{array}$ & Porcentaje \\
Pretest & 3,4 & 0,77 & $39,5 \%$ \\
Postest & 5,5 & 0,57 & $74,4 \%$ \\
\hline
\end{tabular}

Fuente: Elaboracíón propia.

Gráfico 2 - Comparación entre pretest y postest, taller año 2017

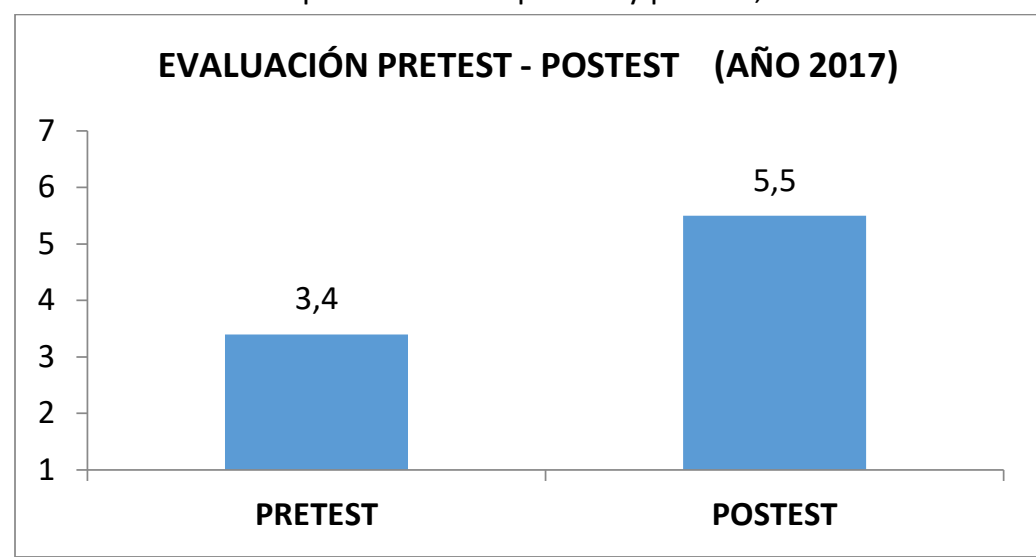

Fuente: Elaboración propia.

Tabla 3 - Prueba paramétrica " $\mathrm{t}$ " de Student, para establecer diferencias entre pretest y postest

\begin{tabular}{|c|c|c|c|}
\hline & Sig. & $\mathbf{T}$ & GI \\
\hline $\begin{array}{c}\text { Notas se ha } \\
\text { considerado } \\
\text { varianza iguales }\end{array}$ & 0,087 & $-8,93$ & 30 \\
\hline
\end{tabular}

Fuente: Elaboracíón propia.

Para 30 grados de libertad, la prueba " $\mathrm{t}$ ", tiene un valor comprendido entre $1,70<\mathrm{t}<1,70$, cuyo intervalo señala que no existe diferencia entre los grupos.

Para este caso, la prueba " $\mathrm{t}$ " arroja un valor comprendido entre, $-8,93<\mathrm{t}<$ 8,93 , como queda lejos del intervalo, podemos asegurar una diferencia importante entre el pretest y postest, debido a la metodología empleada para el taller de Webquest.

Para usar una estadística no paramétrica y compara entre pretest y postest se utiliza la prueba $U$ de Mann-Whitney. 
Tabla 4 - Estadística no paramétrica: Prueba U de Mann-Whitney

\begin{tabular}{cc} 
Estadísticos de contraste & Notas \\
U de Mann-Whitney & 3,5 \\
W de Wilcoxon & 123,5 \\
Z & $-4,72$ \\
\hline
\end{tabular}

Fuente: Elaboracíón propia.

El valor de $-1,96<Z<1,96$, corresponde a muestra grandes, con $n>20$. En esta caso, se obtiene un $-4,72<Z<4,72$, indicando una gran diferencia entre pretest y postest.

En cuanto al producto obtenido en los tres talleres, de acuerdo a los diferentes grupos de trabajo, y según los años 2015, 2016 y 2017, se muestran en la tabla que se señala a continuación

Tabla 5 - Webquest producidas por alumnos del taller durante los años 2015,2016 y 2017

\begin{tabular}{|c|c|c|c|c|}
\hline Año & Grupo & Tema & Nivel & Disciplina \\
\hline 2015 & $1(2)$ & $\begin{array}{l}\text { Ojo humano y sus } \\
\text { enfermedades }\end{array}$ & 3M & Biologia \\
\hline 2015 & $2(2)$ & Fotosíntesis & $19 \mathrm{M}$ & Biologia \\
\hline 2015 & $3(3)$ & $\begin{array}{c}\text { Aplicación de leyes de } \\
\text { Newton }\end{array}$ & Pregrado & Física \\
\hline 2015 & $4(3)$ & Nutrición y salud & $8 \circ B$ & Cs. Nat. \\
\hline 2015 & $5(2)$ & El átomo y la materia & $8 B$ & Cs. Nat. \\
\hline 2015 & $6(2)$ & Disolución en Química & $29 \mathrm{M}$ & Química \\
\hline 2015 & $7(3)$ & $\begin{array}{c}\text { Presión: Principio de } \\
\text { Pascal }\end{array}$ & 3M & Física \\
\hline 2015 & $8(1)$ & Vectores & Pregrado & Física \\
\hline 2015 & $9(1)$ & Webquest las vacunas & 4M & Biologia \\
\hline 2016 & $1(3)$ & $\begin{array}{c}\text { Webquest para } \\
\text { aprendizaje de } \\
\text { expresiones algebraicas }\end{array}$ & $80 B$ & Matemát. \\
\hline 2016 & $2(3)$ & $\begin{array}{l}\text { Estructura y función de } \\
\text { la celula }\end{array}$ & $19 \mathrm{M}$ & Biologia \\
\hline 2016 & $3(3)$ & $\begin{array}{l}\text { Webquest cambio de } \\
\text { materia }\end{array}$ & 70 B & Cs. Nat. \\
\hline
\end{tabular}




\begin{tabular}{|c|c|c|c|c|}
\hline 2016 & $4(3)$ & $\begin{array}{l}\text { Conservación de energia } \\
\text { mecánica }\end{array}$ & 20M & Física \\
\hline 2016 & $5(2)$ & Calentamiento global & 3M & Física \\
\hline 2016 & $6(3)$ & $\begin{array}{l}\text { Bacterias ¿amigas o } \\
\text { enemigas ocultas? }\end{array}$ & 7으 & Cs. Nat. \\
\hline 2016 & $7(3)$ & $\begin{array}{c}\text { Desarrollando } \\
\text { habilidades del } \\
\text { pensamento científico }\end{array}$ & 5B-4M & Cs.Nat. \\
\hline 2017 & $1(2)$ & Nutrición y salud & $8 \div B$ & Cs. Nat. \\
\hline 2017 & $2(3)$ & $\begin{array}{l}\text { Métodos de } \\
\text { electrización }\end{array}$ & $8 \div B$ & Cs. Nat. \\
\hline 2017 & $3(2)$ & Escalas termometricas & $19 \mathrm{M}$ & Física \\
\hline 2017 & $4(3)$ & $\begin{array}{c}\text { Formas de propagación } \\
\text { del calor }\end{array}$ & $8 \div B$ & Cs. Nat. \\
\hline 2017 & $5(2)$ & Teorema de Pitagoras & $2 \circ \mathrm{M}$ & Matemát. \\
\hline 2017 & $6(2)$ & $\begin{array}{c}\text { Movimiento retilíneo } \\
\text { uniforme }\end{array}$ & 20M & Física \\
\hline 2017 & $7(3)$ & $\begin{array}{l}\text { La matéria y sus } \\
\text { transformaciones }\end{array}$ & 7으 $B$ & Cs. Nat. \\
\hline 2017 & $8(1)$ & $\begin{array}{l}\text { La célula unidad } \\
\text { funcional de seres vivo }\end{array}$ & 2이 & Biologia \\
\hline
\end{tabular}

Fuente: Elaboracíón propia.

De los resultados de la tabla anterior, producto de las contribuciones de cada grupo se presenta dos gráficos, uno según sus disciplinas y el otro según el nivel.

Gráfico 3 - Porcentaje de contribuciones según la disciplina

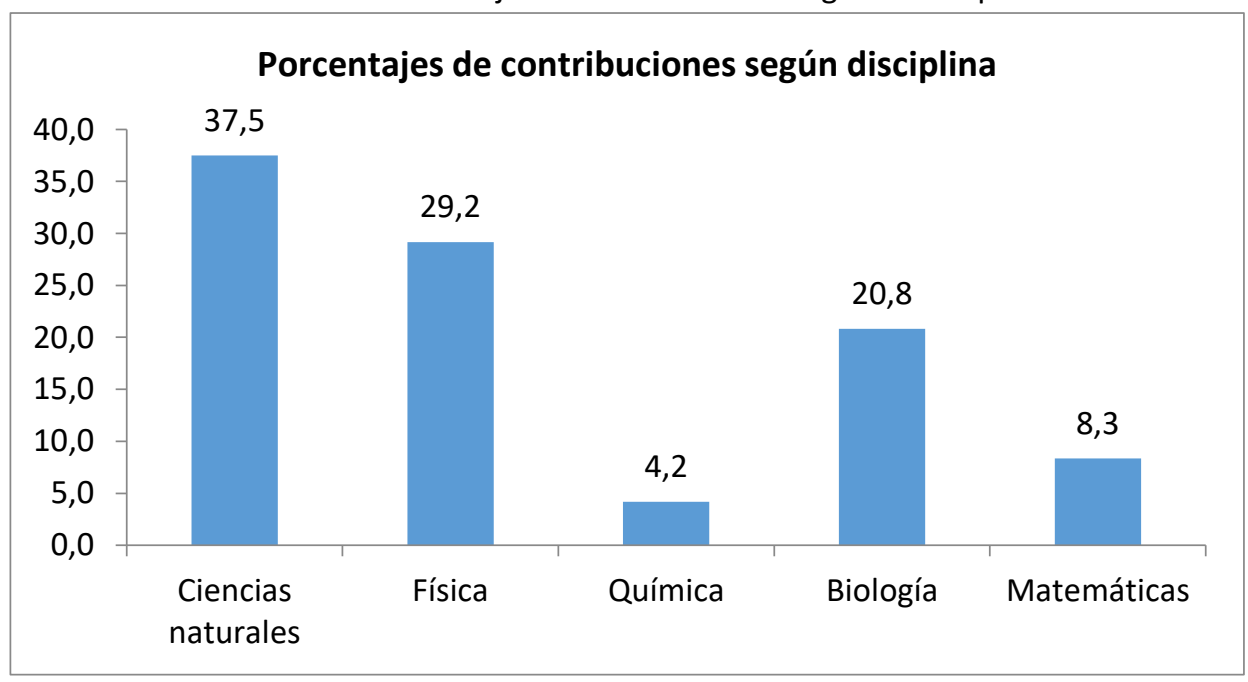

Fuente: Elaboracíón propia. 
Gráfico 4 - Porcentaje de contribuciones de acuerdo al nivel de aplicación

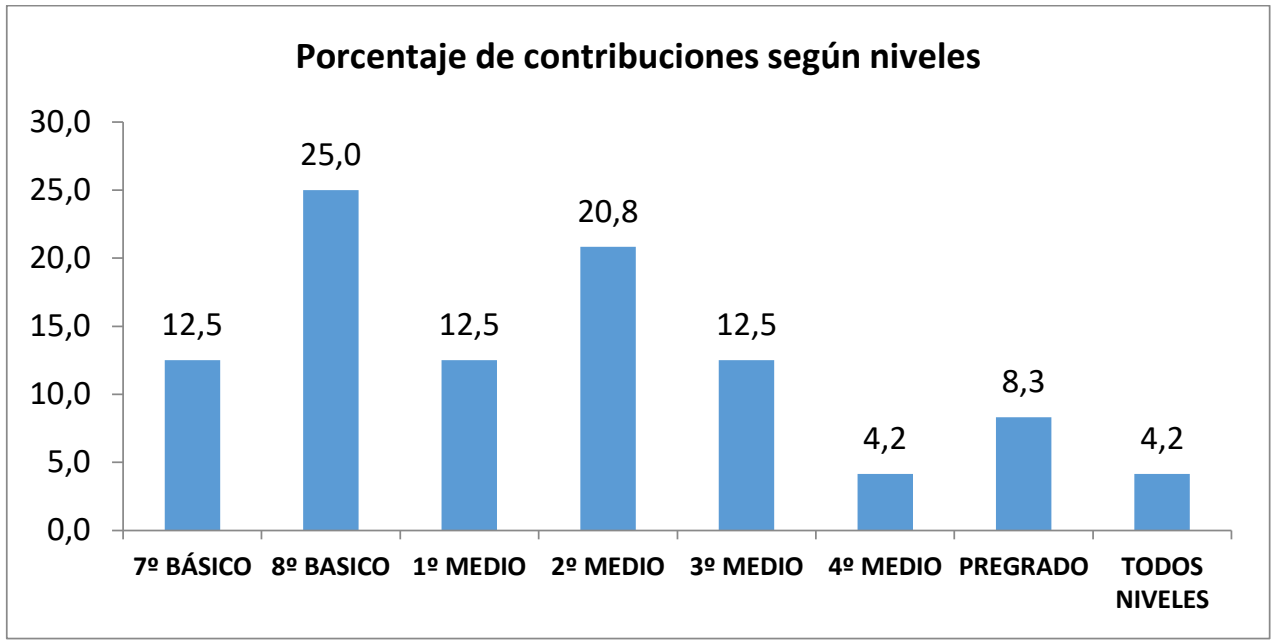

Fuente: Elaboracíón propia.

A continuación se muestra una de las portadas de los productos de Webquest.

Webquest sobre tipos de propagación del calor

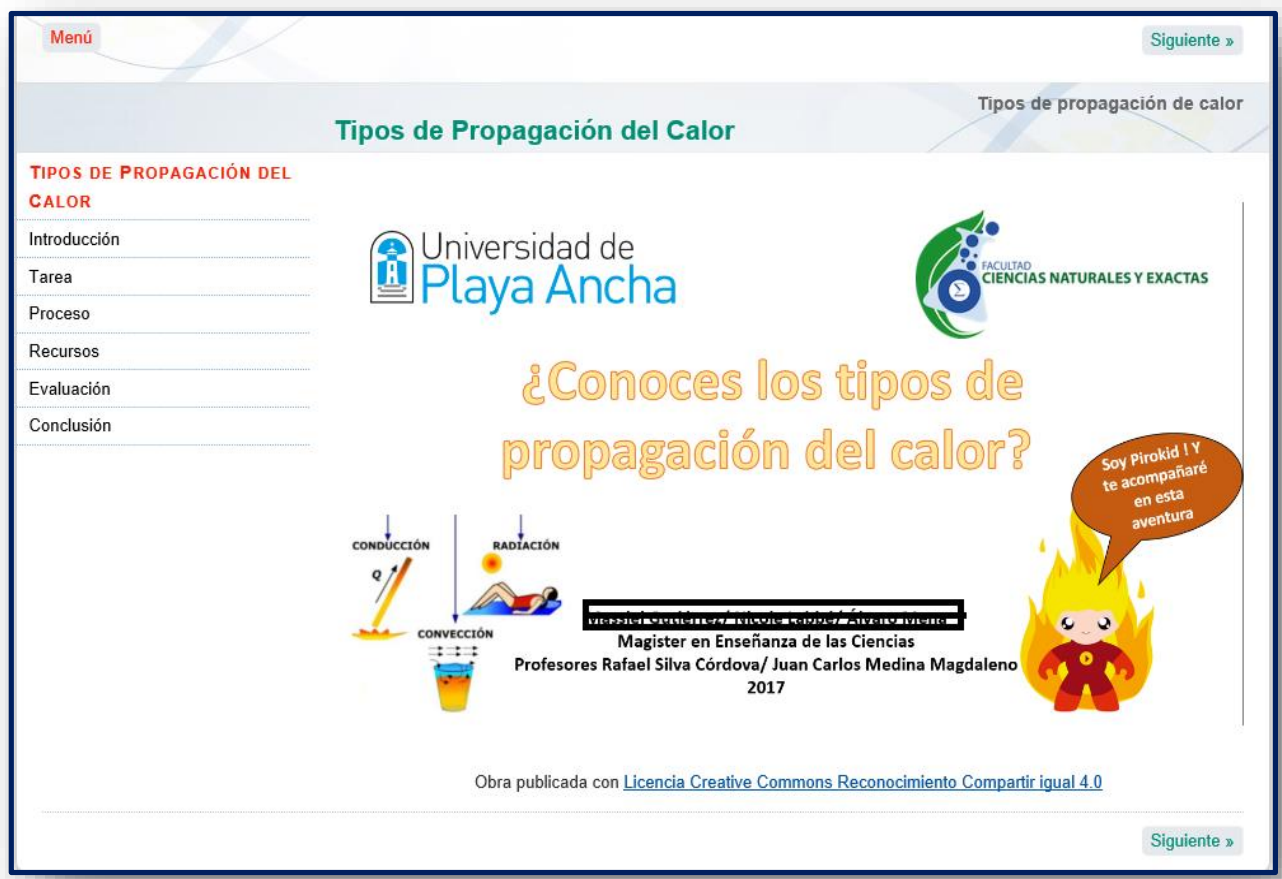

Fuente: Elaboracíón propia. 


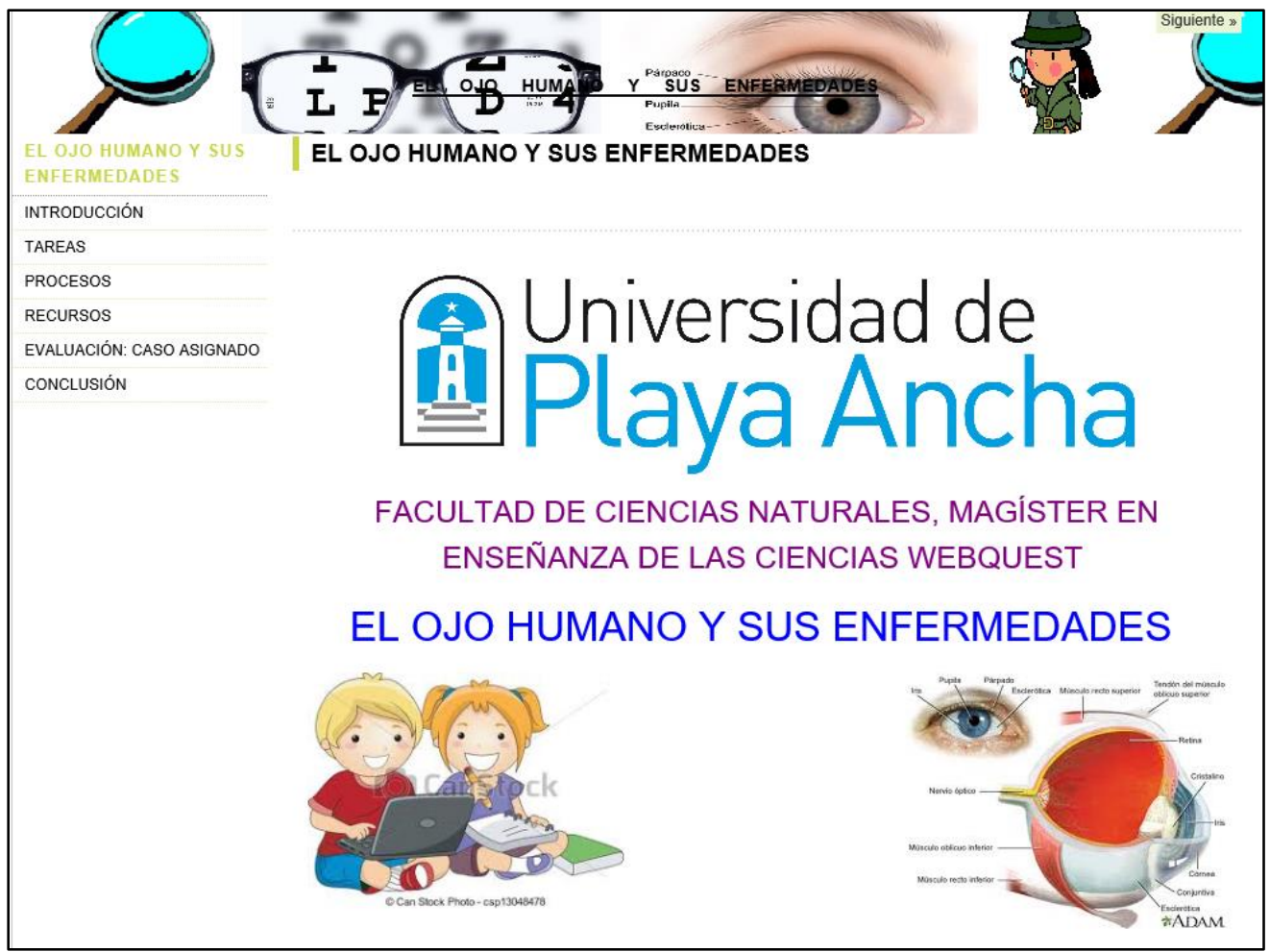

Fuente: Elaboracíón propia.

\section{CONCLUSIONES}

A la luz de los resultados obtenidos, se puede aseverar que la metodología de enseñanza del taller "Uso de las TIC en la enseñanza de las Ciencias", focalizada en la capacitación del uso de la Webquest como recurso didáctico para la enseñanza de las ciencias se puede concluir:

(1) El taller de uso de TIC para la enseñanza de las ciencias, en todas sus versiones $(2015,2016,2017)$ han cumplido rigurosamente con la planificación de que estas se han propuesto.

(2) El taller en esta últimas tres propuesta ha tenido en cada año un rendimiento muy satisfactorio en todo sus participantes, con rendimiento de 5,9 (año 2015), 6,1 (año 2016) y 5,9 (año 2017), basada en la Webquest para la promoción de aprendizaje significativo está bien organizada y planificada, que no presenta mayores inconvenientes en su aplicación.

(3) La metodología experimental de la investigación nos indica un avance importante entre el pretest y postest, de 3,4 a 5,5 según escala de nota de 1,0 a 7,0 . Esta diferencia es avalada por la prueba paramétrica " $\mathrm{t}$ " de Student y por la muestra no paramétrica $U$ de Mann-Whitney.

(4) En relación al aprendizaje se puede inferir que los alumnos en general lograron aprendizaje significativo sobre el uso de la Webquest como recurso didáctico para la enseñanza de las ciencias.

En cuanto a la producción del recurso didáctico para la enseñanza media se puede concluir: 
(1) Se diseñaron 24 Webquest, en diferentes contenidos desde la enseñanza básica hasta dos en el nivel de pregrado de enseñanza superior.

(2) De acuerdo a las disciplina se construyeron un 37,5\% en ciencias naturales, 29,2 \% em Física, 4,4\% en Química, 20,8\% en Biología y 8,3 \% en Matemáticas.

(3) Según el nivel se elaboraron un 37,5\% para el nivel básico, un $50 \%$ para la el nivel medio, un $8,3 \%$ para el nivel superior y un $4,2 \%$ que abarca todos los niveles.

En consecuencia, ha sido una experiencia académica importante y exitosa, que ha dado respuesta a las preguntas de investigación, con el cumplimiento de sus objetivos propuestos. 


\title{
THE WEBQUEST AS A DIDACTIC RESOURCE FOR THE PROMOTION OF SIGNIFICANT LEARNING IN NATURAL AND EXACT SCIENCES
}

\begin{abstract}
The Webquest is a type of didactic activity based on constructivist assumptions of the teaching - learning process. This research, in the context of innovation, proposes a didactic experience for three groups of teachers, to design, develop and implement webquest for the teaching of science. It is guided by a guided indagatory methodology that promotes meaningful learning and improves academic performance. The methodological teaching proposal is theoretically based on the significant learning of Ausubel, on cognitive developments of Piaget, zone of proximal development of Vygotsky and the cooperative work of Jonhson and Jonhson. The research is experimental, essentially quantitative, with an exploratory, descriptive study. The results ensure an important advance between the pretest and posttest of each work group, with a tangible product that can be used in the science classrooms.
\end{abstract}

KEYWORDS: Webquest. Meaningful learning. Academic performance. Assimilation. Inquiry teaching methodology. 


\section{AGRADECIMIENTOS}

Destacamos con especial gratitud al Programa de Magister en Enseñanza de la Ciencias (acreditado 7 años), de la Universidad de Playa Ancha, por su apoyo y estímulo a la investigación en enseñanza de las ciencias.

\section{REFERENCIAS}

AIELLO, M., BARTOLOME, A., y WILLEN, C. Evaluando 5 años de semipresencialidad en Comunicación Audiovisual. Comunicación presentada en el $3 r$ Congreso Internacional "Docencia Universitaria y Innovación", Girona, Julio 2004.

AUSUBEL, D. The acquisition and retention of knowledge: a cognitive view. Dordrecht: Kluwer Academic Publishers. 212p., 2000.

BARTOLOME, A. Blended Learning: Conceptos Básicos. Pixel-Bits, Revista de Medios y Educación, № 23, pp 7-20. Universidad de Barcelona, 2004. Disponible en https://recyt.fecyt.es/index.php/pixel/article/viewFile/61237/37251 Acceso el 2 Ene 2019.

BARTOLOME, A. Universidades en la Red. ¿Universidad presencial o virtual? En Crítica, V52, N896, pp. 34-38, 2002.

CABERO, J. La aplicación de las TIC, ¿esnobismo o necesidad educativa?, 2003. Disponible en: http://reddigital.cnice.mec.es/1/firmas/firmas cabero ind.html.

COATEN, N. Blended e-learning. Suplemento del boletín de educaweb | ISSN: 1578-5793. 6 de octubre de 2003. Disponible en: https://www.educaweb.com/esp/servicios/monografico/formacionvirtual/11810 76-a.html\# Acceso en 2 de enero 2019.

COLLINS, B. Tele-learning in a digital world. The future of distance learning. London. International Thomson Computer Press, 1996.

KHAN, B. Web-Based Training: An introducción. Nueva Jersey. Education Tecnolology Publications, pp. 5-12, 2001.

LEAO, M. B. C., BARTOLOME, A. R. Multiambiente de aprendizagem: a integração da sala de aula com os laboratórios experimentais e de multimeios. Revista Brasileira de Tecnología Educacional. Anos XXX/XXI, Nos 159/160. p.75-80. Marzo 2003. 
MOREIRA, M. A.. A teoria da aprendizagem significativa e sua implementação em sala de aula. Brasília: Editora da UnB. 2006. 185p.

MOREIRA,M.A. Aprendizaje significativo: teoría y práctica. Madrid: VISOR. 100 , 2000.

MURPHY, P. 2003. The hybrid strategy: Blending face-to-face with virtual instruction to improve large section courses. University of California Regents. Teaching, Learning, and Technology Center. Disponible en: http://www.uctltc.org/news/2002/12/feature print.html. Acceso el 7 de septiembre 2010.

PASCUAL, Ma P. 2003. El Blended learning reduce el ahorro de la formación on-line pero gana en calidad. Educaweb, 69. 6 de octubre de 2003. http://www.educaweb.com/esp/servicios/monografico/formacionvirtual/118110 8.asp Acceso en 2 de enero 2019.

SILVA, R., LOPEZ, E. Laboratorio Virtual para un curso de Física Experimental. IX Encuentro de Informática y Computación en Educación. Universidad las Américas. Santiago, Chile, Agosto 2001.

SILVA, R. , LOPEZ, E. El uso de una herramienta virtual en el laboratorio de Física. Ponencia presentada en XI Encuentro de Física Regional Norte. V Reunión Internacional Andina de Física. Universidad de Antofagasta. Chile. 10 al 12 de Octubre 2001.

SILVA, R., LOPEZ, E. Un curso de Física experimental en una plataforma virtual. Ponencia presentada en REF XII. Duodécima Reunión de Educación en Física. Universidad Nacional San Martín. Buenos Aires. Argentina. 20 al 22 de Septiembre de 2001.

SILVA, R., LOPEZ, E. Metodología Activa para la Enseñanza de la Física con apoyo de e-Learning. Visiones Científicas. Volumen 7, № 1,2, año 2004.

SILVA, R., LOPEZ, E. Modelo metodológico didáctico para la enseñanza de la física experimental ambientado en Blended Learning. Visiones Científicas. Volumen 8, № 1, año 2009. 
SILVA, R, LOPEZ, E. 2010 Incorporación de la Webquest al proceso enseñanza aprendizaje de la Física Moderna. Revista Visiones Científicas Volumen 9, № 1. Pág. 25-34, año 2010.

Recebido: 11 mar. 2019

Aprovado: 16 jun. 2020

DOI: $10.3895 /$ rbect.v13n2.9820

Como citar: CORDOVA, R. S.; DONOSO, E. L.; MAGDALENO, J. C. M. La webquest como recurso didactico para la promocion de aprendizaje significativo en ciencias naturales y exactas. Revista Brasileira de Ensino de Ciência e Tecnologia, Ponta Grossa, v.13, n. 2, p. 1-17, mai./ago. 2020. Disponível em: <https://periodicos.utfpr.edu.br/rbect/article/view/9820>. Acesso em: XXX

Correspondência: Rafael Silva Cordova - rsilvacordova@gmail.com

Direito autoral: Este artigo está licenciado sob os termos da Licença Creative Commons-Atribuição 4.0 Internacional. 